\title{
Effect of familial diabetes status and age at diagnosis on type 2 diabetes risk: a nation-wide register-based study from Denmark
}

\author{
Omar Silverman-Retana ${ }^{1,2,3}$ (D) Adam Hulman ${ }^{1,2,3} \cdot$ Jannie Nielsen $^{4,5} \cdot$ Claus T. Ekstrøm $^{6} \cdot$ Bendix Carstensen $^{7}$. \\ Rebecca K. Simmons ${ }^{1} \cdot$ Lasse Bjerg $^{1,2,3} \cdot$ Luke W. Johnston $^{1,2,3} \cdot$ Daniel R. Witte $^{1,2,3}$
}

Received: 14 November 2019 / Accepted: 31 January 2020 / Published online: 19 February 2020

(C) Springer-Verlag GmbH Germany, part of Springer Nature 2020

\begin{abstract}
Aims/hypothesis We assessed whether the risk of developing type 2 diabetes and the age of onset varied with the age at diabetes diagnosis of affected family members.

Methods We performed a national register-based open cohort study of individuals living in Denmark between 1995 and 2012. The population under study consisted of all individuals aged 30 years or older without diagnosed diabetes at the start date of the cohort (1 January 1995) and who had information about their parents' identity. Individuals who turned 30 years of age during the observation period and had available parental identity information were also added to the cohort from that date (open cohort design). These criteria restricted the study population mostly to people born between 1960 and 1982. Multivariable Poisson regression models adjusted for current age and highest educational attainment were used to estimate incidence rate ratios (IRRs) of type 2 diabetes.

Results We followed 2,000,552 individuals for a median of 14 years (24,034,059 person-years) and observed 76,633 new cases of type 2 diabetes. Compared with individuals of the same age and sex who did not have a parent or full sibling with diabetes, the highest risk of developing type 2 diabetes was observed in individuals with family members diagnosed at an early age. The IRR was progressively lower with a higher age at diabetes diagnosis in family members: 3.9 vs 1.4 for those with a parental age at diagnosis of 50 or 80 years, respectively; and 3.3 vs 2.0 for those with a full sibling's age at diagnosis of 30 or 60 years, respectively.

Conclusions/interpretation People with a family member diagnosed with diabetes at an earlier age are more likely to develop diabetes and also to develop it at an earlier age than those with a family member diagnosed in later life. This finding highlights the importance of expanding our understanding of the interplay between genetic diabetes determinants and the social, behavioural and environmental diabetes determinants that track in families across generations. Accurate registration of age at diagnosis should form an integral part of recording a diabetes family history, as it provides easily obtainable and highly relevant detail that may improve identification of individuals at increased risk of younger onset of type 2 diabetes. In particular, these individuals may benefit from closer risk factor assessment and follow-up, as well as prevention strategies that may involve the family.
\end{abstract}

Keywords Clinical science $\cdot$ Epidemiology $\cdot$ Prediction and prevention of type 2 diabetes

Electronic supplementary material The online version of this article (https://doi.org/10.1007/s00125-020-05113-8) cotains peer-reviewed but unedited supplementary material, which is available to authorised users.

Omar Silverman-Retana

omar.silverman@ph.au.dk

1 Department of Public Health, Aarhus University, Building 1260, Barthollins Allé 2, 8000 Aarhus C, Aarhus, Denmark

2 Danish Diabetes Academy, Odense, Denmark

3 Steno Diabetes Center Aarhus, Aarhus University Hospital, Aarhus, Denmark
4 Global Health Section, Department of Public Health, University of Copenhagen, Copenhagen, Denmark

5 Hubert Department of Global Health, Rollins School of Public Health, Emory University, Atlanta, GA, USA

6 Section of Biostatistics, Department of Public Health, University of Copenhagen, Copenhagen, Denmark

7 Clinical Epidemiology Department, Steno Diabetes Center Copenhagen, Gentofte, Denmark 


\section{Research in context}

\section{What is already known about this subject?}

- Family history of diabetes is a strong independent risk factor for developing type 2 diabetes. It has been used as a risk component in several type 2 diabetes screening and risk prediction tools, often defined as a binary indicator

- Familial risk of diabetes depends on the number of family members affected with the disease and the type of family relationship

What is the key question?

- What is the effect of family members' age at diabetes diagnosis on an individual's risk of developing diabetes, and on the age at which they may develop it?

\section{What are the new findings?}

- People with a family member diagnosed with diabetes earlier in middle-age are more likely to develop diabetes and also to develop it at an earlier age than those with a family member diagnosed in later life

\section{How might this impact on clinical practice in the foreseeable future?}

- Accurate registration of age at diagnosis should form an integral part of recording a diabetes family history, as it provides easily obtainable and highly relevant detail that may improve identification of individuals at increased risk of younger onset of type 2 diabetes. In particular, these individuals may benefit from closer risk factor assessment and follow-up, as well as prevention strategies that may involve the family

\section{Abbreviations \\ CPR Central Person Register \\ IRR Incidence rate ratio \\ NDR National Diabetes Register}

\section{Introduction}

Family history of diabetes is a simple risk marker widely used in different diabetes screening and risk prediction tools [1]. It is a convenient and informative risk factor, which provides a lens into both the genetic and social/behavioural determinants of the disease. There are up to 250 known genetic variants for type 2 diabetes risk [2]. However, taken together, these variants only explain around $5 \%$ of the population-wide diabetes risk and less than $15 \%$ of diabetes heritability [3]. Moreover, the strongest known determinants of type 2 diabetes have well-established social and behavioural components: obesity, physical inactivity, diet and low socioeconomic status [4]. Each of these factors clusters in families [5, 6], and can track across multiple generations [7, 8].

A broad body of evidence has shown that parental diabetes increases the risk of diabetes in populations from different ethnic backgrounds two to fourfold [9], and stronger risk estimates have been reported if the mother is the parent diagnosed with diabetes [9]. Furthermore, familial type 2 diabetes risk has been shown to depend on the type of family relationship and the number of family members affected with diabetes [10]. Despite progress on understanding the aetiological details of diabetes heritability, little is known about the effect of family members' age at diabetes diagnosis on an individual's risk of developing diabetes, and on the age at which they may develop it. A deeper understanding of these effects may give greater insight into why an early onset of type 2 diabetes appears to have a worse prognosis [11], and thus stimulate wider use of familial age at diagnosis in clinical and public health practice.

We hypothesised that, in addition to the type of family relationship and the number of family members affected with diabetes, their age at diagnosis would be associated with overall familial diabetes risk. In order to study this hypothesis, we carried out a national register linkage study covering the entire Danish population.

\section{Methods}

This register-based open cohort study included all individuals living in Denmark at any point between 1995 and 2012 (Fig. 1). Our study population consisted of all individuals aged 30 years or older without diagnosed diabetes at the entry into the cohort, and who had information about their parents' identity $(N=$ $2,018,916)$. We refer to this population as index individuals as we followed them with regard to the outcome, and they each served as a starting point for identification of parental and sibling relations. Index individuals were followed from 1 January 1995, or date of cohort entry, until the date of emigration or death, or until 31 December 2012.

Data from national registers were linked using the unique personal registration number (Central Person Register [CPR] number), which all Danish residents are given at birth or immigration. Registers included The Danish Civil Registration 


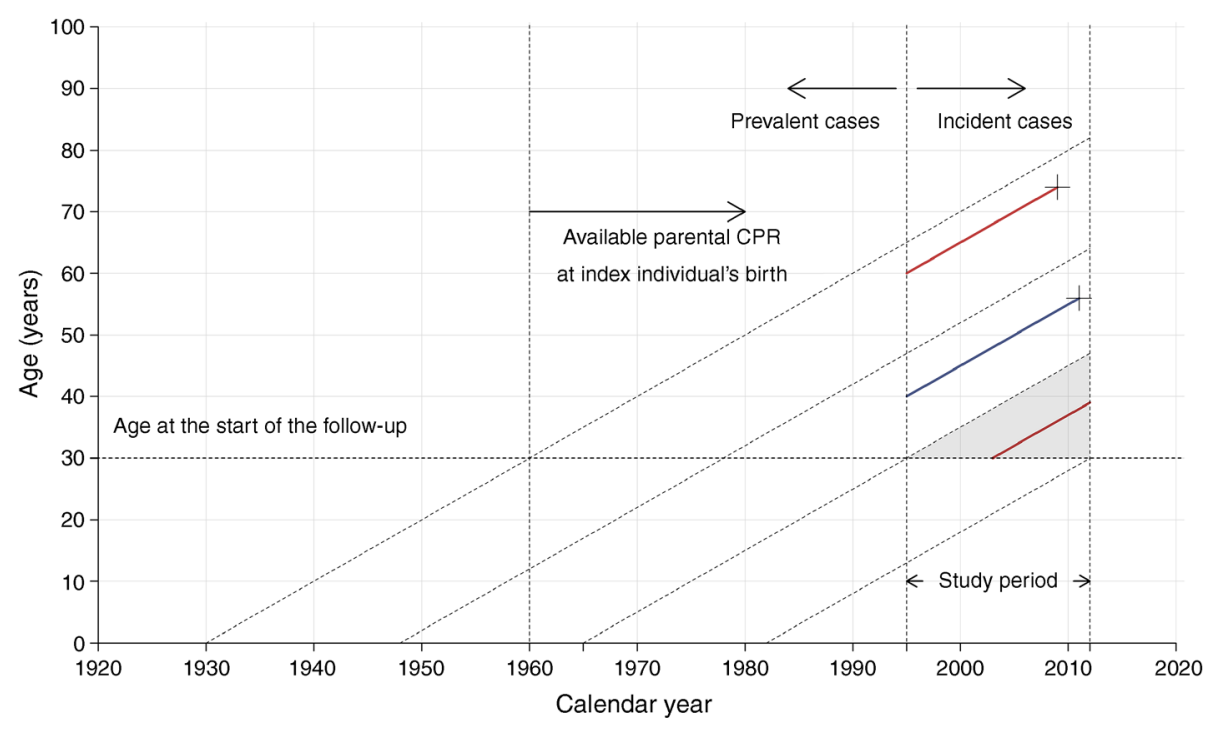

Fig. 1 Lexis diagram showing the population under study. Lifelines (solid coloured diagonal lines) represent three hypothetical cohort members' follow-up: two index individuals (siblings) and their mother. The cross indicates the diagnosis of type 2 diabetes. The blue line represents a male index individual who entered the cohort at the age of 40 years in 1995 and developed type 2 diabetes in 2011 after 16 years of follow-up. The upper solid red line shows his mother's follow-up, which started at the age of 60 years in 1995, followed by the development of type 2 diabetes in 2009, at the age of 74 years. She is not an index individual as she was born before parental information was recorded. She

System [12], the Danish National Diabetes Register (NDR) [13], the Educational Attainment Register (www.dst.dk/en/ Statistik/dokumentation/documentationofstatistics/highesteducation-attained) and the Fertility Database (www.dst.dk/en/ Statistik/dokumentation/documentationofstatistics/fertilitydatabase). The Danish Data Protection Agency approved the study.

Complete reciprocal reference between both legal parents' and children's CPR numbers is available for children born in Denmark from 1960 and onwards [12]. We used the Fertility Database to identify family relationships. Full siblings were defined by identifying individuals sharing the same paternal and maternal CPR numbers; those only sharing one CPR number, either paternal or maternal, were defined as half siblings.

Outcome The outcome of interest was the incidence of type 2 diabetes in the index individual (first record of diabetes in the follow-up period). The NDR combines data from the National Patient Register, the National Health Insurance Service Register and the Register of Medicinal Product Statistics to classify individuals as having diabetes [13]. The earliest registration date of one the following criteria was used to define diabetes cases, as well as the date of disease onset in the NDR: (1) diagnosis of diabetes in the National Patient Register; (2) record of chiropody for patients with diabetes; (3) the fifth blood glucose measurement recorded within a 1 year window; contributes to her children's exposure by marking 14 person-years of their follow-up as not exposed to maternal diabetes and then by marking 3 additional person-years as exposed to maternal diabetes. The lowest solid diagonal red line represents a younger full sibling who entered the cohort when she turned 30 years in 2003, and who was followed and remained diabetes free until the end of follow-up in 2012. This younger sibling is a separate index individual, exposed to the same maternal diabetes status as her brother. She also contributes by marking 9 person-years of her brother's risk as not exposed to a full sibling with diabetes

(4) the second blood glucose measurement per year in 5 consecutive years; (5) second purchase of oral glucoselowering drugs recorded within 6 months; or (6) second purchase of prescribed insulin [13]. This algorithm identified $96 \%$ of the population with diabetes and had a positive predictive value of $89 \%$ [13]. The completeness and ascertainment of the register has been previously validated [14]. Given that in the NDR it is not possible to distinguish between type 1 diabetes and type 2 diabetes cases, we considered 30 years as the age at diagnosis cut-off value to classify index individuals as having a type 2 diabetes diagnosis. This criterion has shown an adequate discriminatory ability for the exclusion of type 1 diabetes cases [15].

Exposure We defined the following time-updated variables: (1) parental diabetes status as binary variables (yes/no); (2) full or half sibling diabetes status as categorical variables that included an indicator for having no full or half siblings $(1=$ having no siblings, $2=$ having siblings without diabetes and $3=$ having siblings with diabetes). Sibling diabetes status definition (full or half) used the earliest date of diabetes diagnosis among each type of sibling (full or half). These variables were updated at the date of each new diagnosis in the NDR. We additionally defined variables for the age at diabetes diagnosis of family members. For all analyses, the reference group consisted of individuals who did not have half siblings and whose parents and full siblings did not have diabetes. 
Covariates Sociodemographic characteristics of index individuals included age at entry to the study and the highest educational attainment achieved during the study period (completing less than 10 years, between 10 and 15 years, or more than 15 years of education) [16]. The reference group for the educational attainment indicator were individuals who completed between 10 and 15 years of education.

Statistical analysis We performed a complete case analysis. The descriptive analysis reported medians (interquartile range) for continuous variables, and frequencies for categorical or binary variables. Participants' follow-up (risk time) was cut into 1-year band intervals by calendar year (1995-2012). Incidence rates for the occurrence of diabetes were estimated with multivariable adjusted Poisson regression models stratified by sex, using log-person-time as the offset variable (natural logarithm). Poisson models included the following covariates: current age, family members' time-updated diabetes status and the highest educational attainment. We included interaction terms to quantify the excess risk for individuals with diabetes in both parents and/or full siblings, relative to the additional effect of each parent or full sibling. Half sibling interaction terms were not included in the models as some of the family combinations showed largely indeterminate estimates (e.g. father $\times$ full siblings $\times$ maternal half siblings). The incidence rate of type 2 diabetes for a 50-year-old individual is presented as a measure of absolute risk for the reference group. This age is used only to provide a general reference for the absolute incidence rate of diabetes in the Danish population. All relative rates are comparisons with people of the same age and sex (without a family member with diabetes at that age). We have chosen the age of 50 years as it broadly represents the start of the age range where type 2 diabetes incidence increases markedly and becomes an important clinical and public health concern.

We fitted a second set of models to assess the effect of the age at diabetes diagnosis of both parents and full siblings on individuals' risk of type 2 diabetes. We restricted the cohort to index individuals with family members who had a date of diabetes registration after 1995 (familial diabetes incident cases), as diagnoses before this date may not have an accurate date of registration. Natural cubic spline effects on index individuals' current age and family member age at diabetes diagnosis were used in these models. From the 'same parental age at diagnosis' models, we additionally calculated age differences in reaching a $2 \%$ and $4 \%$ cumulative incidence of type 2 diabetes. This analysis was performed to quantify the relationship between parental age at diabetes diagnosis and type 2 diabetes onset in the index individual.

As families may have more than one child, our analyses may include more than one index individual per family. For instance, in a family composed of both parents and three children older than 30 years, there would be a familial dependence of the outcome between the individuals. In order to address this familial correlation between observations, we used a clustered covariance matrix by family to compute robust standard errors and $95 \% \mathrm{CI}$.

All statistical analyses were performed using $\mathrm{R}$ version 3.5.1 [17]. Data management and processing was done with the data.table (version 1.11.8) [18] and dplyr (version 0.7.8) [19] packages, the Epi (version 2.32) [20] and sandwich (version 2.5-0) [21] packages were used for modelling, and the ggplot2 (version 3.1.0) [22] package was used for data visualisation.

\section{Results}

After exclusion of individuals with missing information on education ( $n=18,364)$, the study population comprised $2,000,552$ individuals in $1,107,915$ families. During 24,034,059 person-years, 76,633 index individuals developed type 2 diabetes. Median follow-up time was 14 years and the median age at entry to the study was 30 years. Men and women had similar sociodemographic backgrounds and familial diabetes indicators (Table 1). However, a larger percentage of women $(42 \%)$ had completed more than 15 years of education compared with men $(31 \%)$.

\section{Effect of familial diabetes status on incident diabetes risk The} estimated diabetes incidence rate for 50-year-old men and women with no family members with diabetes, and who completed 10-15 years of education, was 4.4/1000 personyears and 3.2/1000 person-years, respectively. Figure 2 displays the associations between exposure to familial diabetes for different family members separately and in combination. Individuals who had either one parent or one full sibling with diabetes had a twofold elevated risk of developing type 2 diabetes compared with individuals who had no family members with diabetes (Fig. 2). Type 2 diabetes risk for individuals without full sibling relations lies between the risk of those individuals who had full siblings without type 2 diabetes (reference group) and those individuals with a full sibling with type 2 diabetes (see electronic supplementary material [ESM] Table 1).

We found a substantial elevation of type 2 diabetes incidence rate ratios (IRRs) when both parents had diabetes $\left(\mathrm{IRR}_{\text {women }} 4.00 ; 95 \%\right.$ CI 3.73, 4.28; $\mathrm{IRR}_{\text {men }} 3.87 ; 95 \% \mathrm{CI}$ $3.65,4.11)$, compared with individuals who had no family members with diabetes. Furthermore, the risk was even higher if the index individuals' mother and full sibling both had diabetes $\left(\right.$ IRR $_{\text {women }} 4.62 ; 95 \%$ CI 4.24, 5.04; IRR $_{\text {men }} 4.40$; $95 \%$ CI 4.09, 4.74). However, the combined estimates were significantly smaller than the product of the two estimates. Unadjusted interaction effects for different familial diabetes combinations, including half siblings, can be found in the ESM Tables 1-5. 
Table 1 Index individuals' sociodemographic background and familial diabetes indicators stratified by sex

\begin{tabular}{|c|c|c|}
\hline Indicator & $\begin{array}{l}\text { Men } \\
\mathrm{N}=1,042,996\end{array}$ & $\begin{array}{l}\text { Women } \\
\mathrm{N}=957,556\end{array}$ \\
\hline \multicolumn{3}{|c|}{ Index individuals' sociodemographic characteristics and diabetes status } \\
\hline Follow-up (years) & $14.1(7.6-17.0)$ & $14.1(7.5-17.0)$ \\
\hline Age at the start of the follow-up (years) & $30.0(30.0-35.5)$ & $30.0(30.0-34.5)$ \\
\hline \multicolumn{3}{|l|}{ Highest educational attainment } \\
\hline Less than 10 years & $222,143(21.3)$ & $166,109(17.3)$ \\
\hline Between 10 and 15 years & $499,842(47.9)$ & $389,244(40.6)$ \\
\hline More than 15 years & $321,011(30.8)$ & $402,203(42.0)$ \\
\hline Index individuals with type 2 diabetes & $44,252(4.2)$ & $32,381(3.4)$ \\
\hline Index individuals' age at diabetes diagnosis (years) & $48.6(42.4-54.5)$ & $46.0(39.3-53.2)$ \\
\hline \multicolumn{3}{|l|}{ Familial diabetes status } \\
\hline Father with diabetes & $164,976(15.8)$ & $150,757(15.7)$ \\
\hline Father's age at diabetes diagnosis (years) & $64.8(58.3-71.5)$ & $64.4(58.0-70.9)$ \\
\hline Mother with diabetes & $147,466(14.1)$ & $131,521(13.7)$ \\
\hline Mother's age at diabetes diagnosis (years) & $64.6(57.6-71.4)$ & $64.2(57.3-70.9)$ \\
\hline Number of full siblings & $1(1-2)$ & $1(1-2)$ \\
\hline \multicolumn{3}{|l|}{ Full sibling diabetes status } \\
\hline No full siblings & $195,910(18.8)$ & $176,960(18.5)$ \\
\hline Full sibling(s) without diabetes & $788,474(75.6)$ & $728,745(76.1)$ \\
\hline Full sibling(s) with diabetes & $58,612(5.6)$ & $51,851(5.4)$ \\
\hline Full sibling age at diabetes diagnosis (years) & $43.5(35.1-50.4)$ & $43.1(34.7-50.1)$ \\
\hline \multicolumn{3}{|l|}{ Paternal half sibling diabetes status } \\
\hline No paternal half siblings & $928,429(89.0)$ & $846,598(88.4)$ \\
\hline Paternal half sibling(s) without diabetes & $108,442(10.4)$ & $105,099(11.0)$ \\
\hline Paternal half sibling(s) with diabetes & $6125(0.6)$ & $5859(0.6)$ \\
\hline Paternal half sibling age at diabetes diagnosis (years) & $40.2(30.6-47.7)$ & $40.2(30.3-47.6)$ \\
\hline \multicolumn{3}{|l|}{ Maternal half sibling diabetes status } \\
\hline No maternal half siblings & $953,374(91.4)$ & $871,065(91.0)$ \\
\hline Maternal half sibling(s) without diabetes & $84,975(8.1)$ & $82,035(8.6)$ \\
\hline Maternal half sibling(s) with diabetes & $4647(0.4)$ & $4456(0.5)$ \\
\hline Maternal half sibling age at diabetes diagnosis (years) & $39.6(30.0-47.0)$ & $39.4(30.2-47.0)$ \\
\hline
\end{tabular}

Continuous variables values are p50 (p25-p75). Binary and categorical variables values are $n(\%)$
Effect of familial age at diagnosis on incident diabetes risk Compared with individuals of the same age and sex who did not have a parent or full sibling with diabetes, the highest risk of developing type 2 diabetes was observed in individuals with family members diagnosed at an early age. The IRR of type 2 diabetes was progressively lower with a higher age at diabetes diagnosis in family members. Type 2 diabetes risk was on average $60 \%$ lower for those with a parent diagnosed at age 80 years compared with those with a parent diagnosed at age 50 years, while it was $40 \%$ lower for those with a full sibling diagnosed at age 60 years compared with those with a full sibling diagnosed at age 30 years (Fig. 3a-c).

For example, compared with individuals of the same age and sex with a mother without diabetes, the IRR of type 2 diabetes for men and women with a mother diagnosed with diabetes at the age of 50 years (Fig. 3a) was 4.47 (95\% CI $4.11,4.87)$ and $3.86(95 \%$ CI $3.57,4.17)$, respectively. The
IRR decreased to $1.45(95 \%$ CI $1.36,1.55)$ for men and to $1.51(95 \%$ CI $1.41,1.62)$ for women with a mother diagnosed with diabetes at the age of 80 years.

Compared with individuals of the same age and sex with a father without diabetes, the IRR for men and women with a father diagnosed with diabetes at the age of 50 years (Fig. 3b) was $3.98(95 \%$ CI 3.56, 4.44) and 3.32 (95\% CI 2.97, 3.70), respectively. The IRR decreased to 1.42 (95\% CI $1.33,1.51)$ for men and to $1.41(95 \%$ CI $1.30,1.54)$ for women with a father diagnosed with diabetes at the age of 80 years.

Compared with individuals of the same age and sex with unaffected full siblings (Fig. 3c), men and women with a full sibling diagnosed with diabetes at the age of 30 years had an IRR of 3.62 (95\% CI 3.34, 3.92) and 3.10 (95\% CI 2.83, 3.39), respectively. The IRR decreased to 1.96 (95\% CI 1.80 to 2.14 ) for men and to $2.07(95 \%$ CI 1.86, 2.29) for women with a full sibling diagnosed with diabetes at the age of 60 years. 


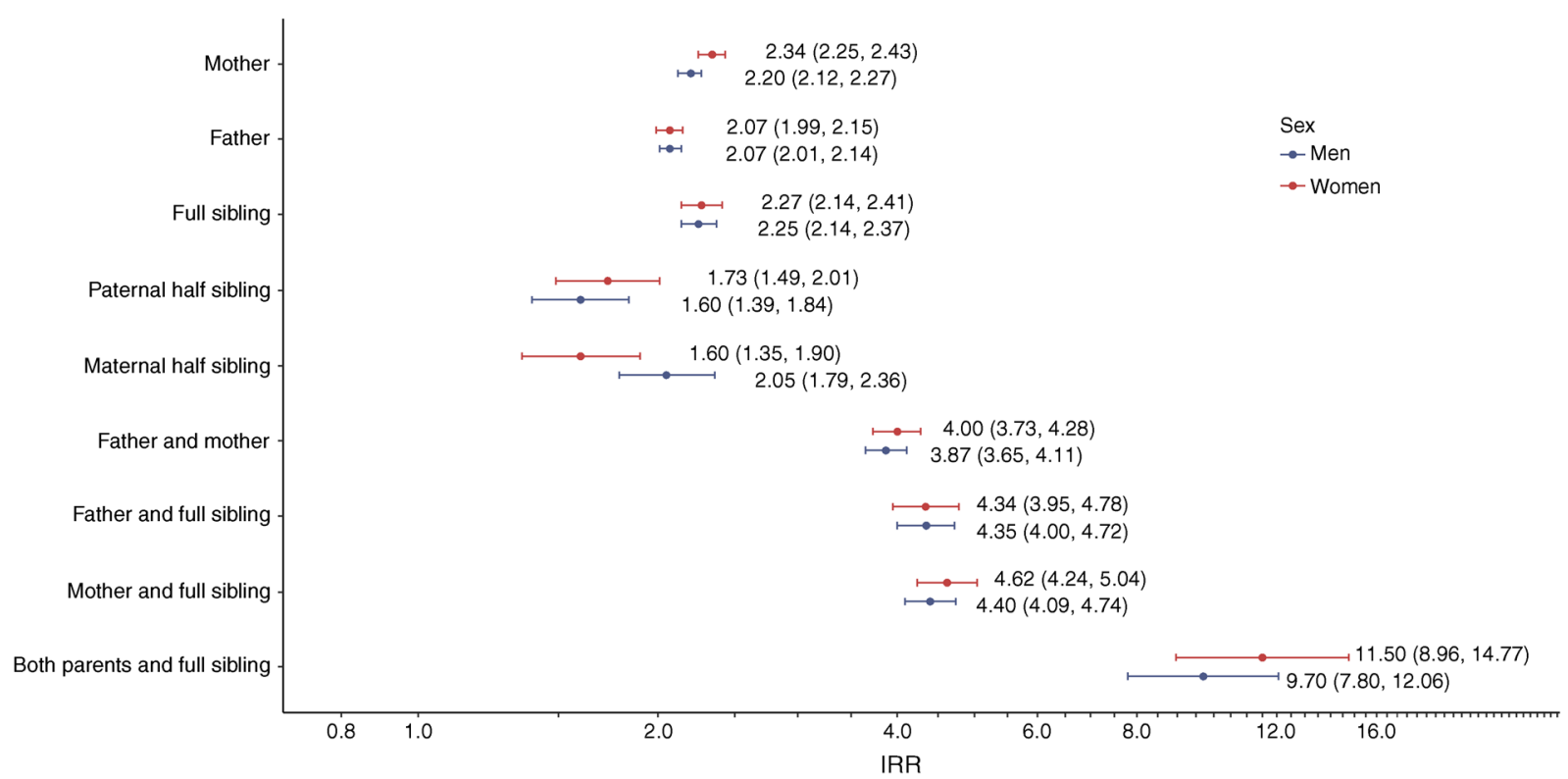

Fig. 2 Sex-stratified familial diabetes IRRs with 95\% CI. Models were adjusted for current age and highest educational attainment. The $x$-axis is on a logarithmic scale (natural log)

Figure 4 shows cumulative incidence of type 2 diabetes over two 20 year age periods for index individuals aged 30 years and 45 years at the start of follow-up (up to age 50 years and 65 years, respectively). Each graph shows cumulative incidence curves for a reference group (not exposed to parental diabetes) and for three different parental diagnosis ages (50, 60 and 70 years). Men and women with a mother without type 2 diabetes barely exceed a $1 \%$ cumulative incidence in the age period 45-65 years (Fig. 4a, b), whereas men with a mother diagnosed with type 2 diabetes at age 50,60 or 70 years reached a $2 \%$ cumulative incidence by the age of 56 , 60 and 64 years, respectively (Fig. 4a). Similar results were observed for women with a mother diagnosed with type 2 diabetes at these three different ages (Fig. 4b).

Men aged 45 years with a father without diabetes reached a $2 \%$ cumulative incidence of type 2 diabetes at the age of

Fig. 3 IRRs by age at diagnosis of diabetes in family members compared with individuals with no affected family member. (a) Mother, (b) father, (c) full siblings. Note that $x$-axes in (a) and (b) (parents' age 50-80 years) are different from the $x$-axis in (c) (full sibling's age $30-60$ years). Models were adjusted for current age and highest educational attainment. The $y$-axis is on a logarithmic scale (natural log)

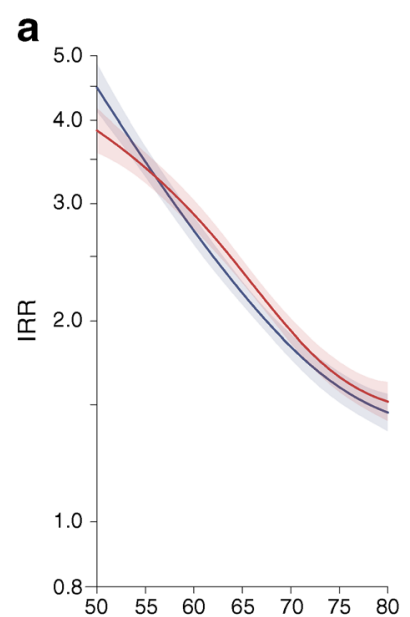

Age at diabetes diagnosis (years)
65 years (Fig. 4c), while the same cumulative incidence level was achieved 11, 8 and 5 years earlier for men with a father diagnosed with diabetes at age 50,60 or 70 years, respectively. Women with a father without type 2 diabetes barely exceed a $1 \%$ cumulative incidence in the age period 45-65 years (Fig. $4 \mathrm{~d}$ ), whereas women with a father diagnosed with type 2 diabetes at age 50,60 or 70 years reached a $2 \%$ cumulative incidence by the age of 58,60 and 64 years, respectively.

\section{Discussion}

Using national registers from Denmark, we showed with high detail how different combinations of exposure to familial diabetes increase an individual's risk of type 2 diabetes. We further showed that the impact of family history of diabetes is b

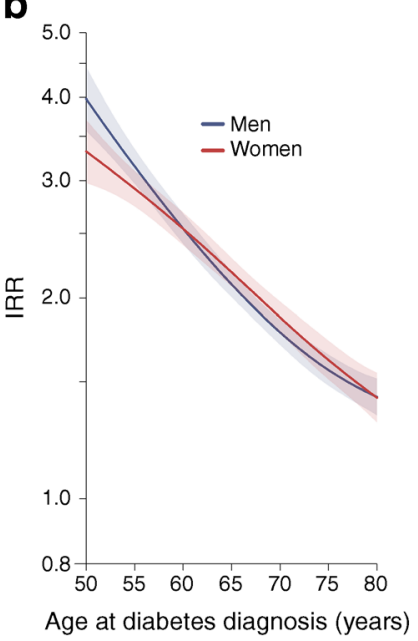

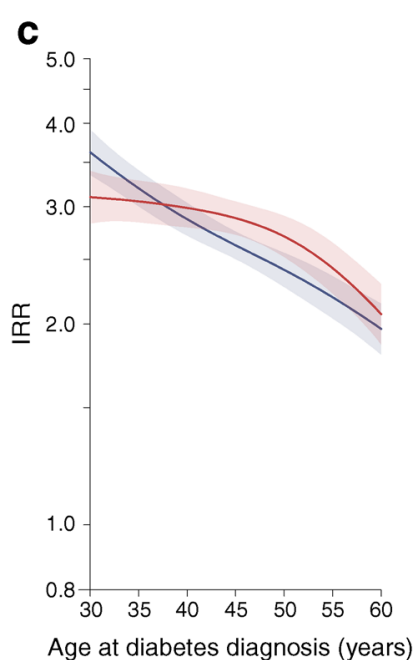


Fig. 4 Cumulative incidence of type 2 diabetes over two 20-year age periods for index individuals aged 30 and 45 years at the start of follow-up (up to age 50 and 65 years, respectively). (a, b) maternal age at type 2 diabetes diagnosis; (c, d) paternal age at type 2 diabetes diagnosis. Blue lines represent men $(\mathbf{a}, \mathbf{c})$ and red lines women $(\mathbf{b}, \mathbf{d})$. Models were adjusted for current age and highest educational attainment. The $y$-axis is on a logarithmic scale (natural $\log$ ) a

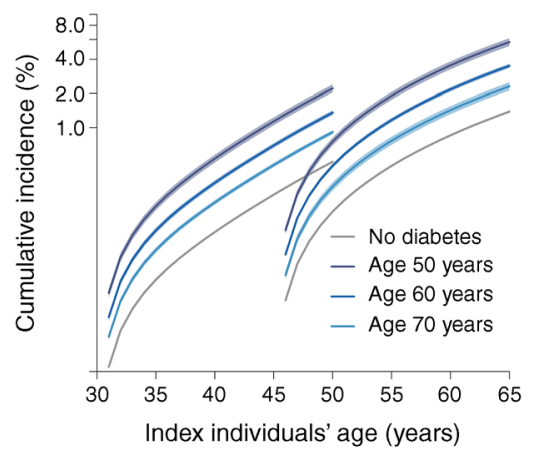

C

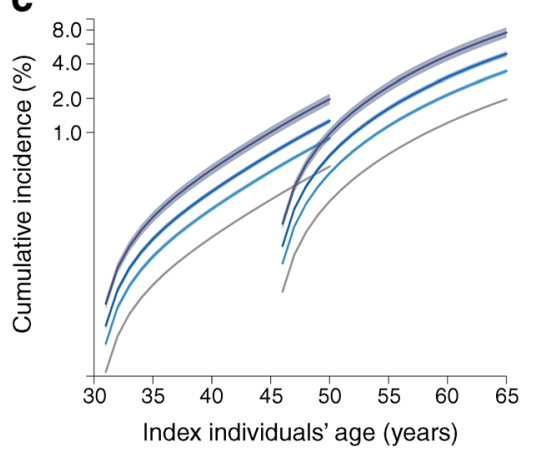

b

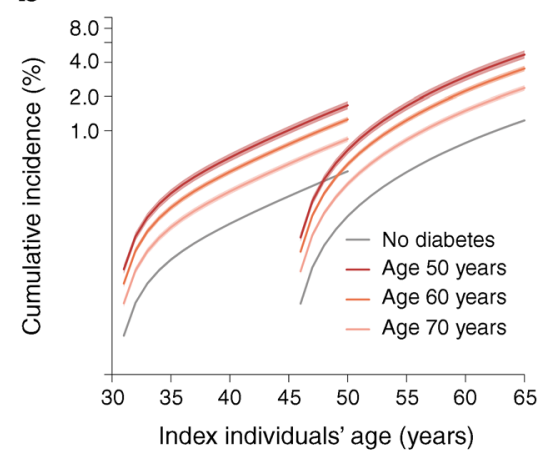

d

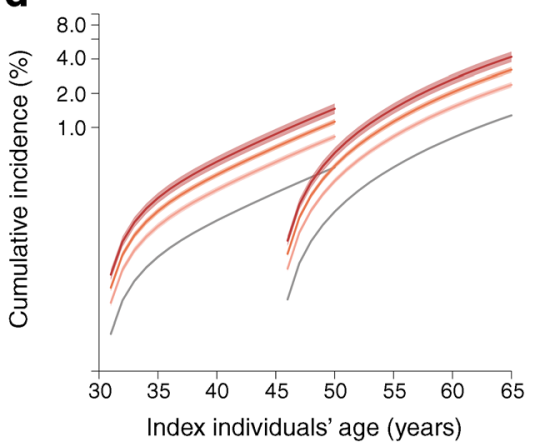

markedly higher when the affected parents or full siblings were diagnosed with the disease at a younger age. Our finding of an overall twofold elevated risk for individuals who have a first-degree relative with diabetes is consistent with previous reports [10, 23, 24]. Moreover, we found similar risk estimates for both paternal and maternal diabetes as opposed to other studies that had reported higher familial risk when the mother was the affected parent [9].

Our study contributes new knowledge about how an individual's risk of type 2 diabetes depends on the age at which a family member is diagnosed with diabetes. The larger risk conferred by parents who are diagnosed with diabetes at a younger age may be the consequence of both a stronger familial genetic susceptibility and a stronger inherited familial diabetogenic environment. Indeed, recent studies suggest that individuals with younger onset of type 2 diabetes experience a more aggressive disease course with regard to the need for insulin [25], complications [26], and higher cardiovascular disease mortality $[11,26]$. The progressive decline of risk for the index individual seen with higher parental age at diabetes diagnosis probably reflects the progressively lower impact of shared heritable (genetic + environmental) factors in favour of individual environmental factors as causes of diabetes at older ages [27].

There is some evidence indicating that genetic determinants of diabetes have a stronger effect in younger people [28]. Previous epidemiological studies have shown that the effect of family history on type 2 diabetes risk is only mediated to a small degree by currently known and measured genetic and lifestyle factors [23, 29]. Thus, a large proportion of variation of diabetes heritability remains unexplained, leaving room for other potential mechanisms (e.g. epigenetic mechanisms, gene-environment interactions) [3, 30, 31], and for effects not fully captured by the current detail and precision of measurements.

We examined whether the risk for individuals with multiple family members with diabetes deviated from the individual contribution of each of those family members. We found that the combined effect was lower than the multiplicative joint effect when both parents, or when the mother and a full sibling, had diabetes. This is likely to be the consequence of the shared exposure to the household environment and, in particular, to social and behavioural mechanisms [5, 6]. Our finding of lower risk levels being conveyed at a given age at diagnosis by a sibling compared with a parent (e.g. at an age of diabetes diagnosis of 50 years, the IRR for having a full sibling with diabetes is $\sim 3$, while for a parent it is $\sim 4$ ), may be the consequence of children in a family primarily learning health behaviours from the parents, and to a lesser degree from siblings. It has been documented that several strong health determinants such as social class, educational attainment and unhealthy behaviours can track across generations [32, 33].

We observed a clear association between a younger age at diagnosis in family members and a higher risk of diabetes in the index individuals (relative risk over a given time window). This association can also be expressed as an age difference in reaching a given level of risk. For example, our analyses show that men with a father with a diabetes diagnosis at age 70 years 
reach a $2 \%$ cumulative incidence of type 2 diabetes 5 years earlier than individuals with a father without type 2 diabetes, while this is 10 years earlier for individuals with a father diagnosed with diabetes at age 50 years. These results show that the two features (age at diagnosis and diabetes risk) are not two separate phenotypes but two sides of the same coin. In fact, genome wide association study (GWAS) analyses in the UK biobank show that age at diabetes diagnosis has a very high heritability index (1.0) [34], driven by a large set of variants on chromosome $6[35,36]$. Many of these variants also feature among the known genetic determinants of type 1 diabetes and type 2 diabetes, among other diseases [37].

Our study is the largest to date investigating how type 2 diabetes onset depends on the age of familial diagnosis of diabetes. A major strength of our open cohort study design is that we followed virtually the entire Danish population born between 1960 and 1982 (from the age of 30 years) for a median of 14 years (up to 17 years). The risk of selection bias is consequently low. The main reason for loss to follow-up was emigration (3.6\% of the cohort); individuals lost to follow-up had a median age of 32 years at emigration and the median year of emigration was the year 2007. Our definition of familial diabetes status used time-updated exposures; therefore, risk time contributions to the exposed and non-exposed groups were calculated with high precision. The exclusion of parents and full siblings with prevalent diabetes (familial diabetes cases registered before 1995, which may not have an accurate date of diagnosis; see Fig. 1) in our age at diagnosis models, avoided an overestimation of type 2 diabetes risk at older familial age of diabetes diagnosis.

The limitations of our study include potential misclassification of the outcome and exposure. The NDR does not distinguish between type 1 diabetes and type 2 diabetes. We addressed this limitation by including only diabetes-free index individuals who were aged 30 years or older in order to focus on type 2 diabetes; however, a low degree of misclassification may still be present [15]. Additionally, the NDR does not capture diabetes cases while they are managed entirely without medication at general practices. Thus, in some cases the registered diagnosis date may be later than the actual clinical diagnosis. A previous study has shown a large increase in the inclusion of patients in the NDR based on use of glucoselowering drugs as the primary ascertainment criterion [14]. This finding might be the result of temporal changes in treatment recommendations. However, these patients quickly met more than one criterion ( $45 \%$ after the first year) and the fraction meeting only one criterion in the long term was about $20 \%$ [13]. Moreover, although these effects are differential over calendar time, they are unlikely to be differential by familial diabetes status at any given time, thus limiting their potential to introduce bias. Another potential source of information bias was the more reliable registration of mothers than fathers for those born before 1960 in Denmark. This potential bias was addressed by including in the analysis only individuals with available linkage to both parents for full sibling definition.

Two main factors restricted the specific birth cohort that could be followed in this analysis and hence the size of our study population (individuals born in Denmark between 1960 and 1973 represented $50 \%$ of our study population and individuals born between 1974 and 1982 represented 25\%). First, parental CPR linkage was only possible for $12 \%$ of individuals born before 1960 and for $40 \%$ of first generation immigrants. Second, our required minimum age of entry to the study (30 years) limits the cohort to individuals born before 1982. This means that for individuals entering the open cohort by turning 30 briefly before or during the observation period (Fig. 1, shaded triangle) most available exposure time falls in the fourth decade of life, an age range with relatively low absolute risk of type 2 diabetes. Consequently, our risk estimates may be conservative. Our study covers a wide age range (from 30 to 80 years), including the decades of life with highest diabetes incidence and prevalence, which both peak around the age of 80 years [38]. Diabetes incidence in people older than 80 years may have a somewhat different aetiology, probably with a less marked contribution of hereditary determinants. In people younger than 30 years, diabetes incidence is largely driven by type 1 diabetes and latent autoimmune diabetes of adulthood (LADA), both of which have a clear familial risk component albeit mediated through other mechanisms. Overall, we consider that generalisability of our results to either side of the studied age range should be done with caution. Finally, since this is a study of the entire population, individual repeated clinical measurements are by definition not available. Therefore, it was not possible to further adjust the models by more proximal determinants of type 2 diabetes such as obesity or physical activity: both characteristics with a strong heritable component $[32,33]$. Moreover, we were not able to assess the relative impact of age of onset of diabetes in a family member against an index individual's risk factors (e.g. BMI) for diabetes. On the basis of previous epidemiological evidence, we could speculate that risk estimates in this study might be more conservative had we been able to adjust the models for these confounders.

In this study, we quantified how familial diabetes risk differs by the age at which the affected family member was diagnosed. We also show how exposure to different combinations of affected family members can increase an individual's risk of type 2 diabetes following a nearly multiplicative pattern. Our findings highlight the importance of expanding our understanding of the interplay between genetic diabetes determinants and the social, behavioural and environmental diabetes determinants that track in families across generations. Accurate registration of age at diagnosis should form an integral part of recording a diabetes family history, as it provides easily obtainable and highly relevant detail that may improve 
identification of individuals at increased risk of developing type 2 diabetes at a younger age. In particular, these individuals may benefit from closer risk factor assessment and follow-up, as well as prevention strategies that may involve the family.

Data availability The data used in this study is owned and managed by Statistics Denmark. In accordance with Danish law and data protection policies, the data used in this study was anonymised, stored and analysed on a secured server. The data can be made available to researchers from research environments pre-approved by Statistics Denmark upon project approval by the Danish Data Protection Agency and Statistics Denmark. Data access can only be granted to researchers and analysts. Researchers can apply for access to these data when the request is approved by the Danish Data Protection Agency: https://www.datatilsynet.dk; the email address for the Danish Data Protection Agency is dt@ datatilsynet.dk.

Funding OSR, AH, LB, LWJ and DRW are funded by the Danish Diabetes Academy, which is, in turn, funded by the Novo Nordisk Foundation. OSR and AH received support provided by the Steno Diabetes Center Aarhus (SDCA), which is partially funded by an unrestricted donation from the Novo Nordisk Foundation. JN is funded by the Independent Research Fund Denmark (DFF - 5053-00263).

Authors' relationships and activities The authors declare that there are no relationships or activities that might bias, or be perceived to bias, their work.

Contribution statement OSR, AH, DRW, JN, CTE, BC, RKS, LB and LWJ made substantial contributions to conception and design of the study, acquisition of data, or analysis and interpretation of data. OSR, AH, DRW, JN, CTE, BC, RKS, LB and LWJ drafted the article or revised it critically for important intellectual content. OSR, AH, DRW, JN, CTE, $\mathrm{BC}, \mathrm{RKS}, \mathrm{LB}$ and LWJ approved the version to be published. DRW is the guarantor of this work.

\section{References}

1. Schwarz PE, Li J, Lindstrom J, Tuomilehto J (2009) Tools for predicting the risk of type 2 diabetes in daily practice. Horm Metab Res 41(2):86-97. https://doi.org/10.1055/s-0028-1087203

2. Langenberg C, Lotta LA (2018) Genomic insights into the causes of type 2 diabetes. Lancet 391(10138):2463-2474. https://doi.org/10. 1016/S0140-6736(18)31132-2

3. Groop L, Pociot F (2014) Genetics of diabetes - are we missing the genes or the disease? Mol Cell Endocrinol 382(1):726-739. https:// doi.org/10.1016/j.mce.2013.04.002

4. Bellou V, Belbasis L, Tzoulaki I, Evangelou E (2018) Risk factors for type 2 diabetes mellitus: an exposure-wide umbrella review of meta-analyses. PLoS One 13(3):1-27. https://doi.org/10.1371/ journal.pone. 0194127

5. Van Zon SKR, Snieder H, Bültmann U, Reijneveld SA (2017) The interaction of socioeconomic position and type 2 diabetes mellitus family history: a cross-sectional analysis of the Lifelines Cohort and Biobank Study. BMJ Open 7(4):1-10. https://doi.org/10.1136/ bmjopen-2016-015275

6. Nielsen J, Bahendeka SK, Whyte SR, Meyrowitsch DW, Bygbjerg IC, Witte DR (2017) Household and familial resemblance in risk factors for type 2 diabetes and related cardiometabolic diseases in rural Uganda: a cross-sectional community sample. BMJ Open 7(9):1-9. https://doi.org/10.1136/bmjopen-2016-015214
7. Portha B, Grandjean V, Movassat $J$ (2019) Mother or father: who is in the front line? Mechanisms underlying the non-genomic transmission of obesity/diabetes via the maternal or the paternal line. Nutrients 11(2):233. https://doi.org/10.3390/nu11020233

8. Miranda-Lora AL, Vilchis-Gil J, Molina-Díaz M, Flores-Huerta S, Klünder-Klünder M (2017) Heritability, parental transmission and environment correlation of pediatric-onset type 2 diabetes mellitus and metabolic syndrome-related traits. Diabetes Res Clin Pract 126: 151-159. https://doi.org/10.1016/j.diabres.2017.02.005

9. Papazafiropoulou AK, Papanas N, Melidonis A, Maltezos E (2017) Family history of type 2 diabetes: does having a diabetic parent increase the risk? Curr Diabetes Rev 13(1):19-25. https://doi.org/ $10.2174 / 1573399812666151022143$

10. Hemminki K, Li X, Sundquist K, Sundquist J (2010) Familial risks for type 2 diabetes in Sweden. Diabetes Care 33(2):293-297. https://doi.org/10.2337/dc09-0947

11. Sattar N, Rawshani A, Franzén S et al (2019) Age at diagnosis of type 2 diabetes mellitus and associations with cardiovascular and mortality risks: findings from the Swedish National Diabetes Registry. Circulation CIRCULATIONAHA.118.037885. https:// doi.org/10.1161/CIRCULATIONAHA.118.037885

12. Pedersen CB (2011) The Danish Civil Registration System. Scand J Public Health 39(7 suppl):22-25. https://doi.org/10.1177/ 1403494810387965

13. Carstensen B, Kristensen JK, Marcussen MM, Borch-Johnsen K (2011) The National Diabetes Register. Scand J Public Health 39(7 Suppl):58-61. https://doi.org/10.1177/1403494811404278

14. Green A, Sortsø C, Jensen PB, Emneus M (2014) Validation of the Danish National Diabetes Register. Clin Epidemiol 7:5-15. https:// doi.org/10.2147/CLEP.S72768

15. Shields BM, Peters JL, Cooper C et al (2015) Can clinical features be used to differentiate type 1 from type 2 diabetes? A systematic review of the literature. BMJ Open 5(11):e009088. https://doi.org/ 10.1136/bmjopen-2015-009088

16. UNESCO Institute for Statistics (2012) International Standard Classification of Education. ISCED 2011. Montreal, Canada

17. R Core Team (2013) R: A language and environment for statistical computing, Vienna, Austria

18. Dowle M, Srinivasan A (2019) data.table: Extension of 'data.frame'. version 1.12.8. https://CRAN.R-project.org/ package $=$ data.table

19. Wickham H, François R, Henry L, Müller K (2019) dplyr: A grammar of data manipulation. $\mathrm{R}$ package version 0.7.6. https://CRAN. R-project.org/package=dplyr

20. Carstensen B, Plummer M (2019) Epi: a package for statistical analysis in epidemiology. R package version 2.40. https://CRAN. R-project.org/package=Epi

21. Berger S, Graham N, Zeileis A (2017) Various versatile variances: an object-oriented implementation of clustered covariances in R. Technical Report 2017-12, Working Papers in Economics and Statistics, Research Platform Empirical and Experimental Economics, Universität Innsbruck. http://EconPapers.RePEc.org/ RePEc:inn:wpaper:2017-12

22. Wickham H (2016) ggplot2: elegant graphics for data analysis. Springer-Verlag New York. https://ggplot2.tidyverse.org

23. Scott RA, Langenberg C, Sharp SJ et al (2013) The link between family history and risk of type 2 diabetes is not explained by anthropometric, lifestyle or genetic risk factors: the EPIC-InterAct study. Diabetologia 56(1):60-69. https://doi.org/10.1007/s00125-012-2715-x

24. Weires MB, Tausch B, Haug PJ, Edwards CQ, Wetter T, CannonAlbright LA (2007) Familiality of diabetes mellitus. Exp Clin Endocrinol Diabetes 115(10):634-640. https://doi.org/10.1055/s2007-984443

25. Zhou K, Donnelly LA, Morris AD et al (2014) Clinical and genetic determinants of progression of type 2 diabetes: a DIRECT study. Diabetes Care 37(3):718-724. https://doi.org/10.2337/dc13-1995 
26. Al-Saeed AH, Constantino MI, Molyneaux L et al (2016) An inverse relationship between age of type 2 diabetes onset and complication risk and mortality: the impact of youth-onset type 2 diabetes. Diabetes Care 39(5):823-829. https://doi.org/10.2337/ dc15-0991

27. Oliynyk RT (2019) Age-related late-onset disease heritability patterns and implications for genome-wide association studies. PeerJ 7:e7168. https://doi.org/10.7717/peerj.7168

28. De Miguel-Yanes JM, Shrader P, Pencina MJ et al (2011) Genetic risk reclassification for type 2 diabetes by age below or above 50 years using 40 type 2 diabetes risk single nucleotide polymorphisms. Diabetes Care 34(1):121-125. https://doi.org/10.2337/ dc10-1265

29. Cornelis MC, Zaitlen N, Hu FB, Kraft P, Price AL (2015) Genetic and environmental components of family history in type 2 diabetes. Hum Genet 134(2):259-267. https://doi.org/10.1007/s00439-0141519-0

30. Gingras V, Hivert MF, Oken E (2018) Early-life exposures and risk of diabetes mellitus and obesity. Curr Diab Rep 18(10). https://doi. org/10.1007/s11892-018-1050-0

31. Franks PW, Merino J (2018) Gene-lifestyle interplay in type 2 diabetes. Curr Opin Genet Dev 50:35-40. https://doi.org/10.1016/ j.gde.2018.02.001

32. Abbasi A, Corpeleijn E, van der Schouw YT et al (2011) Maternal and paternal transmission of type 2 diabetes: influence of diet, lifestyle and adiposity. J Intern Med 270(4):388-396. https://doi.org/ 10.1111/j.1365-2796.2011.02347.x
33. Isomaa B, Forsén B, Lahti K et al (2010) A family history of diabetes is associated with reduced physical fitness in the Prevalence, Prediction and Prevention of Diabetes (PPP)-Botnia study. Diabetologia 53(8):1709-1713. https://doi.org/10.1007/s00125010-1776-y

34. Ge T, Chen CY, Neale BM, Sabuncu MR, Smoller JW (2017) Phenome-wide heritability analysis of the UK Biobank. PLoS Genet 13(4):1-21. https://doi.org/10.1371/journal.pgen.1006711

35. McInnes G, Tanigawa Y, Deboever C et al (2019) Global Biobank Engine: enabling genotype-phenotype browsing for biobank summary statistics. Bioinformatics 35(14):2495-2497. https://doi. org/10.1093/bioinformatics/bty999

36. Global Biobank Engine, Stanford, CA. https://biobankengine. stanford.edu/RIVAS_HG19/coding/INI2976. Accessed 1 Sep 2019

37. Global Biobank Engine, Standford, CA. https://biobankengine. stanford.edu/RIVAS_HG19/variant/6-32626272-C-A. Accessed 1 Sep 2019

38. Carstensen B, Kristensen JK, Ottosen P, Borch-Johnsen K (2008) The Danish National Diabetes Register: trends in incidence, prevalence and mortality. Diabetologia 51(12):2187-2196. https://doi. org/10.1007/s00125-008-1156-Z

Publisher's note Springer Nature remains neutral with regard to jurisdictional claims in published maps and institutional affiliations. 